Tropical Journal of Pharmaceutical Research August 2020; 19 (8): 1605-1610

ISSN: $1596-5996$ (print); 1596-9827 (electronic)

(C) Pharmacotherapy Group, Faculty of Pharmacy, University of Benin, Benin City, 300001 Nigeria.

\title{
Ruscogenin alleviates palmitic acid-induced endothelial cell inflammation by suppressing TXNIP/NLRP3 pathway
}

\author{
Hongtao Liu, Simin Zheng, Hongfei Xiong, Xiaoli Niu* \\ Department of Anesthesia, Second Affiliated hospital of Xi'an JiaoTong University, Xi'an City, Shaanxi Province 710004, China
}

*For correspondence: Email: AXZE90kl@163.com; Tel: +86-29-87679696

Sent for review: 2 June 2020

Revised accepted: 24 July 2020

\begin{abstract}
Purpose: To investigate the involvement of ruscogenin in palmitic acid (PA)-induced endothelial cell inflammation.

Method: Cultured human umbilical vein endothelial cells (HUVECs) were divided into five groups: control (normal untreated cells), PA (cell treated with palmitic acid), and PA + ruscogenin (1, 10, or 30 $\mu M)$. Cell viability and apoptosis rate were determined using MTT (3-(4,5)-dimethylthiahiazo(-z-y1)-3,5di-phenytetrazolium bromide) and flow cytometry assays, respectively. The levels of cytokines, including interleukin-1 $\beta$ (IL-1 $\beta$ ), tumor necrosis factor- $\alpha$ (TNF- $\alpha$ ), intercellular adhesion molecule-1 (ICAM-1), and monocyte chemo-attractant protein-1 (MCP-1) were determined by an enzyme-linked immunosorbent assay. Western blotting and real-time polymerase chain reaction (RT-PCR) were used to evaluate the underlying mechanisms of action.

Results: PA treatment decreased the viability of HUVECs and induced apoptosis ( $p<0.05)$. Ruscogenin attenuated PA-induced cell death in a dose-dependent manner $(p<0.05)$. On the other hand, PA induced an increase in IL-1 $\beta, T N F-\alpha$, ICAM-1, MCP-1, TXNIP (thioredoxin-interacting protein), as well as NLRP3 (nucleotide oligomerization domain-, leucine-rich repeat- and pyrin domain-containing protein 3), all of which were attenuated by ruscogenin $(p<0.05)$.

Conclusion: Ruscogenin alleviates PA-induced endothelial cell inflammation via TXNIP/NLRP3 pathway, thereby providing an insight into new therapeutic strategies to treat cardiovascular diseases.

Keywords: Ruscogenin, Palmitic acid, Endothelial cells, Inflammation, TXNIP, NLRP3, Cardiovascular diseases

This is an Open Access article that uses a fund-ing model which does not charge readers or their institutions for access and distributed under the terms of the Creative Commons Attribution License (http://creativecommons.org/licenses/by/4.0) and the Budapest Open Access Initiative (http://www.budapestopenaccessinitiative.org/read), which permit unrestricted use, distribution, and reproduction in any medium, provided the original work is properly credited.

Tropical Journal of Pharmaceutical Research is indexed by Science Citation Index (SciSearch), Scopus, International Pharmaceutical Abstract, Chemical Abstracts, Embase, Index Copernicus, EBSCO, African Index Medicus, JournalSeek, Journal Citation Reports/Science Edition, Directory of Open Access Journals (DOAJ), African Journal Online, Bioline International, Open-J-Gate and Pharmacy Abstracts
\end{abstract}

\section{INTRODUCTION}

Cardiovascular diseases are the leading cause of death worldwide and involve abnormal endothelial cell inflammation [1]. Inflammation leads to abnormal production of reactive oxygen species (ROS), decreased nitric oxide (NO) levels, and endothelial dysfunction and injury, which also contribute to atherosclerosis [2]. Therefore, inhibiting endothelial inflammation is of great interest to prevent cardiovascular diseases.

Elevation of free fatty acids (FFAs) could induce inflammatory responses and impair vascular reactivity, a known pathogenetic mechanism of 
cardiovascular diseases [3]. PA (palmitic acid) is an abundant FFAs, leading to lipid overload that can induce a harmful inflammatory cascade and plays a key role in several chronic diseases, including atherosclerosis [4]. PA is a ligand of endogenous toll-like receptor-4 (TLR4) and promotes inflammatory cytokine secretion, as well as the production of cytoplasmic messengers including ROS, leading to the development of atherosclerosis [5]. Recently, PA was shown to induce activation of NLRP3 (nucleotide oligomerization domain-, leucine-rich repeat- and pyrin domain-containing protein 3 ) inflammasomes. This eventually leads to the production of active caspase-1 [6] that enhances secretion of proinflammatory interleukin-1 $\beta$ (IL$1 \beta)$ to exacerbate local inflammation [7]. Therefore, PA could induce endothelial inflammation and lead to cardiovascular disease development.

Ruscogenin exerts anti-inflammatory effect against diabetic nephropathy [8] and has been shown to suppress endothelial inflammatory responses in the setting of vascular disorders [9]. However, the effect of ruscogenin on endothelial inflammation and its potential mechanisms remain elusive. In this study, a PA-induced endothelial cell model was used to determine the protective effect and mechanisms of ruscogenin in endothelial inflammation.

\section{EXPERIMENTAL}

\section{Cell culture}

Human umbilical vein endothelial cells (HUVECs) were acquired from the Chinese Academy of Sciences (Shanghai, China) and cultured in Ham's F-12K medium (Upstate Biotechnology, Lake Placid, NY, USA) supplemented with $10 \%$ fetal bovine serum (Upstate Biotechnology) and maintained in a $37{ }^{\circ} \mathrm{C}$ incubator with $5 \% \mathrm{CO}_{2}$.

\section{Cell viability and apoptosis}

Cultured HUVECs were seeded and pre-treated with indicated concentrations of ruscogenin (1, 10 , or $30 \mu \mathrm{M}$ ) for 2 hours, followed by exposure to $500 \mu \mathrm{M}$ PA for another 24 hours. For determination of cell viability, the cells were incubated with $5 \mathrm{mg} / \mathrm{mL}$ MTT (3-(4,5)dimethylthiahiazo(-z-y1)-3,5-di-phenytetrazolium bromide) for 4 hours. After 10 minutes, the absorbance at $450 \mathrm{~nm}$ was determined by a microplate reader (Biotek, Winooski, VT, USA). For determination of apoptosis, the cells were harvested and stained with $5 \mu \mathrm{L} 100 \mu \mathrm{g} / \mathrm{mL}$ propidium iodide for 30 minutes. After incubation with fluorescein isothiocyanate-conjugated annexin $\mathrm{V}$, the cells were analyzed using a flow cytometer (Attune, Life Technologies, Darmstadt, Germany).

\section{Determination of inflammatory cytokines}

Culture medium from HUVECs pre-treated with or without indicated concentrations of ruscogenin $(1,10$, or $30 \mu \mathrm{M})$ were collected and centrifuged, and the supernatant levels of $\mathrm{IL}-1 \beta$, tumor necrosis factor- $\alpha$ (TNF- $\alpha$ ), intercellular adhesion molecule-1 (ICAM-1), and monocyte chemoattractant protein-1 (MCP-1)were determined by a commercial enzyme-linked immunosorbent assay kits (Thermo Fisher Scientific, Waltham, MA, USA).

\section{Real-time PCR}

RNA was extracted from cultured HUVECs with TRIzol (Thermo Fisher Scientific) and reversetranscribed into cDNAs. Real-time PCR was

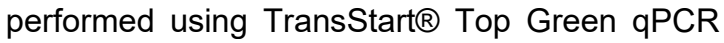
SuperMix (Bio-Rad, Hercules, CA, USA) with glyceraldehyde 3-phosphate dehydrogenase (GAPDH) as a negative control. The primer sequences are shown in Table 1.

Table 1: Primer sequences

\begin{tabular}{ll}
\hline Gene & Sequence \\
\hline NLRP3 & Forward: 5'- \\
& CTCGCATTGGTTCTGAGCTC-3' \\
& Reverse: 5'- \\
& AGTAAGGCCGGAATTCACA-3' \\
TXNIP & Forward: 5'- \\
& GATACCCCAGAAGCTCCTCC-3' \\
& Reverse: 5'- \\
& ACCTCAGTGTAAGTGGGTGG-3' \\
GAPDH & Forward: 5'- \\
& AGTCAGCTCTCTCCTTTCAGG-3' \\
& Reverse: 5'- \\
& TCCACCACCCTGTTGCTGTA-3' \\
\hline
\end{tabular}

\section{Western blot}

Proteins were extracted from HUVECs by radioimmunoprecipitation assay buffer (Thermo Fisher Scientific) and quantified by bicinchoninic protein assay (Thermo Fisher Scientific). Extracted proteins $(40 \mu \mathrm{g})$ were separated with sodium dodecyl sulfate-polyacrylamide gel electrophoresis and transferred to polyvinylidene fluoride membranes. The membranes were blocked in $5 \%$ bovine serum albumin and incubated overnight with the following primary antibodies: thioredoxin-interacting protein (TXNIP), NLRP3 (1:2000, Abcam, Cambridge, UK), and $\beta$-actin (1:3000, Abcam). After incubation with horseradish peroxidaseconjugated secondary antibody, bands were exposed using enhanced chemiluminescent 
detection reagent (Vazyme Biotechnology, Nanjing, China). The signals were analyzed using Image Lab ${ }^{\mathrm{TM}}$ software (Bio-Rad).

\section{Statistical analysis}

Data are presented as mean \pm SEM and were analyzed with GraphPad Prism software 5.0 (GraphPad Inc., San Diego, CA, USA). Multiple groups were compared with one-way analysis of variance. Differences were considered significant at $p<0.05$.

\section{RESULTS}

Ruscogenin attenuated the PA-induced decrease in HUVECs viability

The possible protective effect of ruscogenin for cell viability was first evaluated in HUVECs. The chemical structure of ruscogenin is shown in Figure $1 \mathrm{~A}$. The HUVECs viability under PA treatment was lower than in the untreated control cells (Figure $1 \mathrm{~B}$ ). Pretreatment with ruscogenin dose-dependently attenuated the PA-induced decrease in viability (Figure $1 \mathrm{~B}$ ), suggesting that ruscogenin protected HUVECs from PA-induced cytotoxicity.

A

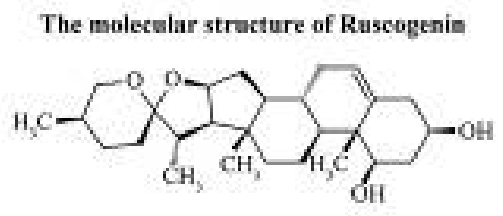

B

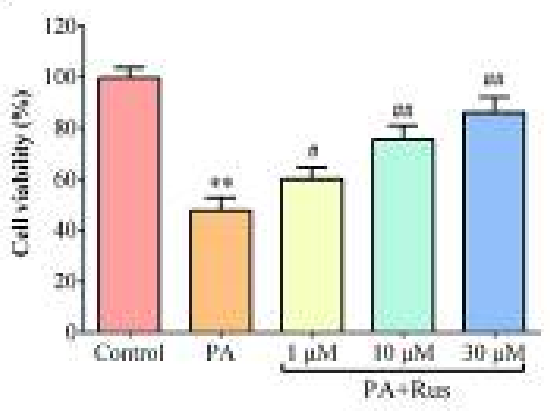

Figure 1: (A) Chemical structure of ruscogenin. (B) Protective effect of ruscogenin on viability in PAinduced HUVECs; ${ }^{*} p<0.01, \# p<0.05, \# \#<0.01$

\section{Ruscogenin blocked PA-induced cell apoptosis}

PA promoted HUVECs apoptosis, but this was dose-dependently ameliorated by ruscogenin pretreatment (Figure 2), confirming its protective effect.
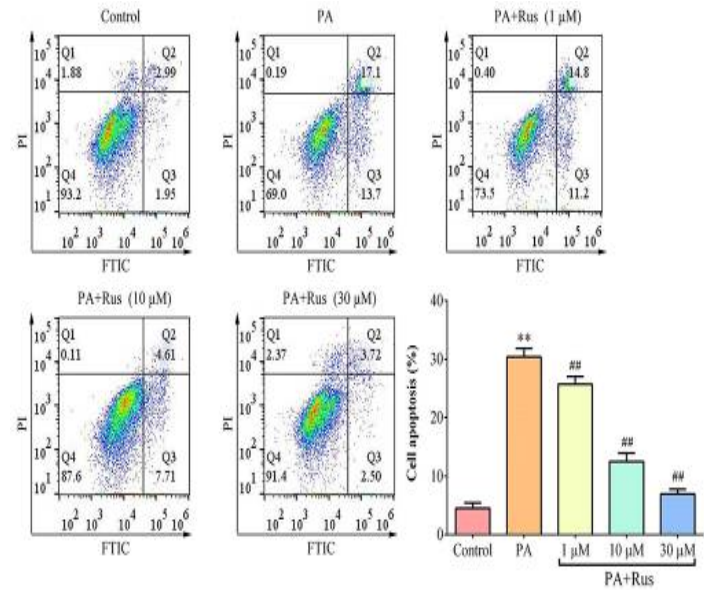

Figure 2: Ruscogenin dose-dependently blocked PAinduced HUVECs apoptosis. ${ }^{* *} p<0.01, \# \# p<0.01$

\section{Ruscogenin alleviated PA-induced inflammation in HUVECs}

To clarify whether ruscogenin could exert an antiinflammatory effect on PA-treated HUVECs, inflammatory cytokine secretion was measured. Exposure of HUVECs to PA produced endothelial cell inflammation as evidenced by the increase in IL-1 $\beta$, TNF- $\alpha$, ICAM-1, and MCP-1 (Figure 3). Ruscogenin dose-dependently decreased the levels of IL- $1 \beta$, TNF- $\alpha$, ICAM- 1 , and MCP-1. These results suggest that ruscogenin effectively alleviated PA-induced inflammation in HUVECs.
A

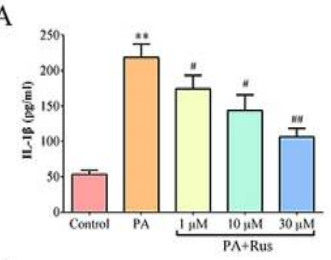

C

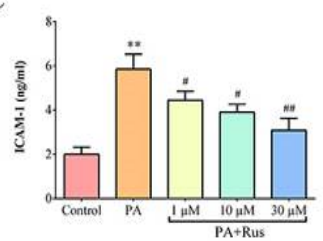

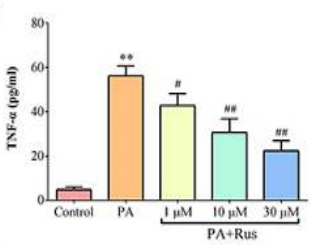

D

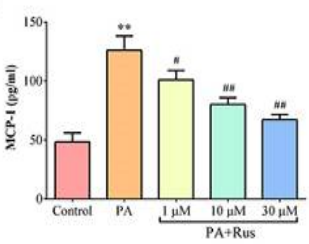

Figure 3: Ruscogenin alleviated PA-induced inflammation in HUVECs. Protective effect of ruscogenin on levels of IL-1 $\beta(A)$, TNF- $\alpha(B)$, ICAM-1 (C) and MCP-1 (D) in PA-induced HUVECs. ${ }^{* *} p<$ $0.01, \# p<0.05, \# \#<0.01$

Ruscogenin inhibited PA-induced activation of TNXIP/NLRP3 signaling

PA-treated HUVECs showed a significant increase in mRNA and protein levels of TNXIP 
(Figure 4). However, pretreatment with ruscogenin dose-dependently decreased all four (Figure $4 \mathrm{C}$ ). These results indicate that ruscogenin inhibited PA-induced activation of the TNXIP/NLRP3 pathway.
A

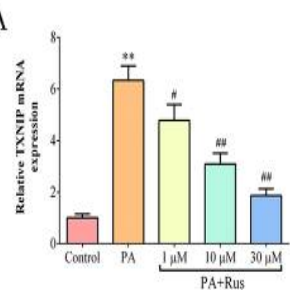

$\mathrm{C}$

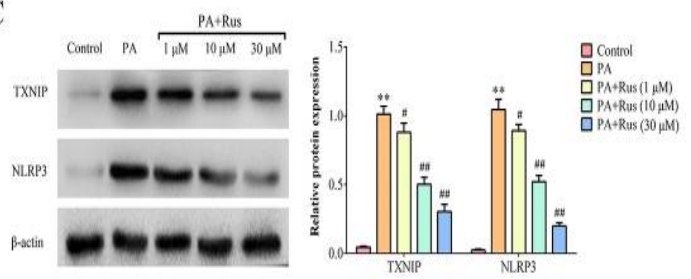

Figure 4: Ruscogenin inhibited PA-induced activation of TNXIP/NLRP3 signaling. (A) Effect of ruscogenin on TXNIP mRNA levels in PA-induced HUVECs. (B) Effect of ruscogenin on NLRP3 mRNA levels in PAinduced HUVECs. (C) Effect of ruscogenin on TXNIP and NLRP3 protein levels in PA-induced HUVECs; ${ }^{* *} p$ $<0.01, \# p<0.05, \# p<0.01$

\section{DISCUSSION}

Ruscogenin, a major bioactive steroid sapogenin, is found in the roots of the traditional Chinese herb, Ophiopogon japonicus, that has been shown to alleviate lipopolysaccharide-induced lung inflammation [10], inhibit oxidative stress and inflammatory diseases [11]. Since endothelial inflammation is considered a hallmark of cardiovascular diseases, the effect of ruscogenin on endothelial inflammation and its possible protective effect against cardiovascular diseases were investigated in this study.

Endothelial cells generally undergo apoptosis after exposure to elevated PA through stimulation of the mitogen-activated protein kinase (MAPK) pathway [12].

PA induces endothelial dysfunction, decreases cell viability, and enhances apoptosis of HUVECs, and used here as a model to investigate mechanisms involved in eicosapentaenoic acid-mediated atherogenesis [13]. The results confirm that PA decreases HUVECs viability and promotes their apoptosis. Ruscogenin has been shown to reduce lipopolysaccharide-induced endothelial cell apoptosis [14]. The present findings demonstrated that ruscogenin exerted an anti- cytotoxic effect in PA-induced HUVECs as evidenced by decreased apoptosis and increased cell viability.

PA can induce endothelial cell apoptosis by enhancing TNF- $\alpha$ expression and ROS accumulation [15]. Moreover, IL-1 $\beta$ and TNF- $\alpha$ function as pro-inflammatory cytokines, and ICAM-1 and MCP-1 function as adhesion molecules that promote endothelial inflammation [16]. Therefore, in line with a previous study that showed PA induced endothelial inflammation by increasing IL-1 $\beta$, TNF- $\alpha$, ICAM-1, and MCP-1 [17], endothelial inflammation by $\mathrm{PA}$ was also demonstrated in this study.

Ruscogenin can reportedly exert antiinflammatory effects by decreasing TNF- $\alpha$, ICAM-1, and MCP-1 [8,9,14]. We also showed that pretreatment with ruscogenin dosedependently inhibited PA-induced inflammation and promoted a suppressive effect on IL-1 $\beta$, TNF- $\alpha, \quad$ ICAM-1 and MCP-1 secretion. ROS accumulation and reduced nitric oxide levels are major contributors to endothelial dysfunction [18]. PA-induced ROS accumulation and low nitric oxide, aggravate endothelial inflammation [17], and the role of ruscogenin on ROS and nitric oxide accumulation in PA-induced HUVECs should be investigated in future studies.

The NLRP3 inflammasome is an important innate immune system component that is activated in vascular inflammation and exacerbates inflammation by enhancing IL-1 1 [19]. ROS accumulation leads to the dissociation of TXNIP from thioredoxin and promotes the binding of TXNIP to NLRP3, thus activating the NLRP3 inflammasome [20]. Therefore, inhibition of TXNIP could serve as a therapeutic strategy for inactivating inflammasomes in the setting of cardiovascular diseases [21]. Consistent with a previous report that TXNIP/NLRP3 was activated in PA-treated HUVECs [21], we observed significant increases in TXNIP and NLRP3 in PAinduced HUVECs. Ruscogenin has been shown to suppress TXNIP/NLRP3 inflammasome activation to ameliorate cerebral ischemia [22].

The results also demonstrate that TXNIP and NLRP3 were dose-dependently decreased in PAinduced HUVECs pretreated with ruscogenin. Collectively, these results indicate that ruscogenin might suppress inflammatory cytokine secretion and NLRP3 inflammasome activation to ameliorate PA-induced cytotoxicity in HUVECs. MAPK and TLR4-mediated nuclear factor (NF)-KB signaling are also involved in PAinduced endothelial inflammation in HUVECs $[23,24]$, and ruscogenin could also regulate these pathways [9-11,14]. Whether MAPK and NF-kB 
pathways are directly involved in ruscogeninmediated endothelial inflammation in PA-induced HUVECs needs to be further investigated.

\section{CONCLUSION}

The findings of this study show that ruscogenin can increases cell viability but decreases the apoptosis of PA-induced HUVECs. The likely mechanism of action is the suppression of the secretion of inflammatory cytokines through inactivation of TXNIP/NLRP3 inflammasome pathways, thus ameliorating PA-triggered endothelial dysfunction. These findings suggest that ruscogenin may be an effective therapeutic agent for cardiovascular diseases.

\section{DECLARATIONS}

\section{Conflict of interest}

No conflict of interest is associated with this work.

\section{Contribution of authors}

We declare that this work was done by the authors named in this article and all liabilities pertaining to claims relating to the content of this article will be borne by the authors. Hongtao Liu and Xiaoli Niu designed the study, supervised the data collection, and analyzed the data; Simin Zheng interpreted the data and prepared the manuscript for publication, and Hongfei Xiong supervised the data collection, analyzed the data, and reviewed the draft of the manuscript. All authors read and approved the final manuscript.

\section{Open Access}

This is an Open Access article that uses a funding model which does not charge readers or their institutions for access and distributed under the terms of the Creative Commons Attribution License (http://creativecommons.org/licenses/by/ 4.0) and the Budapest Open Access Initiative (http://www.budapestopenaccessinitiative.org/rea d), which permit unrestricted use, distribution, and reproduction in any medium, provided the original work is properly credited.

\section{REFERENCES}

1. Li J-K, Liu X-D, Shen L, Zeng W-M, Qiu G-Z. Natural plant polyphenols for alleviating oxidative damage in man: Current status and future perspectives. Trop $J$ Pharm Res 2016; 15(5): 1089.
2. Widlansky ME, Gokce N, Keaney JF, Vita JA. The clinical implications of endothelial dysfunction. J Am Coll Cardiol 2003; 42(7): 1149-1160.

3. Ghosh A, Gao L, Thakur A, Siu PM, Lai CWK. Role of free fatty acids in endothelial dysfunction. J Biomed Sci 2017; 24(1): 50.

4. Calder PC. Long chain fatty acids and gene expression in inflammation and immunity. CurR Opin Clin Nutr 2013; 16(4): 425-433.

5. Seneviratne AN, Sivagurunathan B, Monaco C. Toll-like receptors and macrophage activation in atherosclerosis. Clin Chim Acta; Int J Clin Chem 2012; 413(1-2): 3-14.

6. Wen $H$, Gris $D$, Lei $Y$, Jha $S$, Zhang L, Huang $M T$, Brickey WJ, Ting JP. Fatty acid-induced NLRP3-ASC inflammasome activation interferes with insulin signaling. Nat Immunol 2011; 12(5): 408-415.

7. Kamo $N$, Ke B, Ghaffari $A A$, Shen $X-d$, Busuttil RW, Cheng G, Kupiec-Weglinski JW. ASC/caspase-1/IL-1 $\beta$ signaling triggers inflammatory responses by promoting HMGB1 induction in liver ischemia/reperfusion injury. Hepatology 2013; 58(1): 351-362.

8. Lu HJ, Tzeng TF, Liou SS, Da Lin S, Wu MC, Liu IM. Ruscogenin ameliorates diabetic nephropathy by its anti-inflammatory and anti-fibrotic effects in streptozotocin-induced diabetic rat. BMC Complem Altern M 2014; 14: 110.

9. Huang YL, Kou JP, Ma L, Song JX, Yu BY. Possible mechanism of the anti-inflammatory activity of ruscogenin: role of intercellular adhesion molecule-1 and nuclear factor-kappaB. J Pharm Sci 2008; 108(2): 198-205.

10. Sun Q, Chen L, Gao M, Jiang W, Shao F, Li J, Wang J, Kou J, Yu B. Ruscogenin inhibits lipopolysaccharideinduced acute lung injury in mice: Involvement of tissue factor, inducible NO synthase and nuclear factor (NF)KB. Int Immunopharm 2012; 12(1): 88-93.

11. Lin YN, Jia R, Liu YH, Gao Y, Wang LL, Kou JP, Yu BY. Ruscogenin suppresses mouse neutrophil activation: Involvement of protein kinase A pathway. J Steroid Biochem 2015; 154: 85-93.

12. Jiang H, Liang C, Liu X, Jiang Q, He Z, Wu J, Pan X, Ren $Y$, Fan M, Li M et al. Palmitic acid promotes endothelial progenitor cells apoptosis via p38 and JNK mitogenactivated protein kinase pathways. Atherosclerosis 2010; 210(1): 71-77.

13. Lee CH, Lee SD, Ou HC, Lai SC, Cheng YJ. Eicosapentaenoic acid protects against palmitic acidinduced endothelial dysfunction via activation of the AMPK/eNOS pathway. Int J Mol Sci 2014; 15(6): 1033410349.

14. Wu Y, Wang Y, Gong S, Tang J, Zhang J, Li F, Yu B, Zhang $Y$, Kou J. Ruscogenin alleviates LPS-induced pulmonary endothelial cell apoptosis by suppressing TLR4 signaling. Biomed Pharmacother 2020; 125: 109868.

15. Jiang HL, Zeng YH, Bai L, WANG GH, Hu AM. Palmitic acid induced vascular endothelial cell apoptosis contributes to the high severity of coronary artery

Trop J Pharm Res, August 2020; 19(8): 1609 
disease in the Uygur population. Biomed Res 2017; 28(2): 982-986

16. Liu Y, Davidson BP, Yue $Q$, Belcik $T$, Xie A, Inaba $Y$, McCarty OJT, Tormoen GW, Zhao Y, Ruggeri ZM et al. Molecular imaging of inflammation and platelet adhesion in advanced atherosclerosis effects of antioxidant therapy with NADPH oxidase inhibition. Circ Cardiovasc Imag 2013; 6(1): 74-82.

17. Zhao $W, W u$ C, Li S, Chen $X$. Adiponectin protects palmitic acid induced endothelial inflammation and insulin resistance via regulating ROS/IKK beta pathways. Cytokine 2016; 88: 167-176.

18. Kawashima S, Yokoyama M. Dysfunction of endothelial nitric oxide synthase and atherosclerosis. Arterioscl, Throm, Vasc Biol 2004; 24(6): 998-1005.

19. Duewell P, Kono H, Rayner KJ, Sirois CM, Vladimer G, Bauernfeind FG, Abela GS, Franchi L, Nunez G, Schnurr $M$ et al. NLRP3 inflammasomes are required for atherogenesis and activated by cholesterol crystals. Nature 2010; 464(7293): 1357-1361.

20. Mohamed IN, Hafez SS, Fairaq A, Ergul A, Imig JD, ElRemessy $A B$. Thioredoxin-interacting protein is required for endothelial NLRP3 inflammasome activation and cell death in a rat model of high-fat diet. Diabetologia 2014; 57(2): 413-423.

21. He B, Zhang B, Wu F, Wang L, Shi X, Qin W, Lin Y, Ma $S$, Liang J. Homoplantaginin Inhibits Palmitic Acidinduced Endothelial Cells Inflammation by Suppressing TLR4 and NLRP3 Inflammasome. J Cardiovasc Pharm 2016; 67(1): 93-101.

22. Cao G, Jiang $N$, Hu Y, Zhang $Y$, Wang G, Yin $M$, Ma $X$, Zhou K, Qi J, Yu B et al. Ruscogenin Attenuates Cerebral Ischemia-Induced Blood-Brain Barrier Dysfunction by Suppressing TXNIP/NLRP3 Inflammasome Activation and the MAPK Pathway. Int $J$ Mol Sci 2016; 17(9).

23. Batumalaie K, Amin MA, Murugan DD, Sattar $M Z$, Abdullah NA. Withaferin A protects against palmitic acidinduced endothelial insulin resistance and dysfunction through suppression of oxidative stress and inflammation. Sci Rep2016; 6: 27236.

24. Shi $X$, Wang S, Luan $H$, Tuerhong $D$, Lin $Y$, Liang J, Xiong Y, Rui L, Wu F. Clinopodium chinense Attenuates Palmitic Acid-Induced Vascular Endothelial Inflammation and Insulin Resistance through TLR4-Mediated NFkappa $B$ and MAPK Pathways. Am J Chinese Med 2019; 47(1): 97-117. 